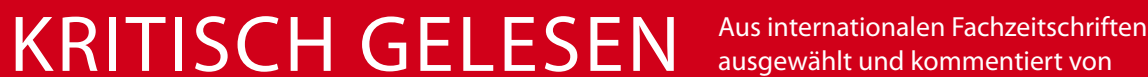

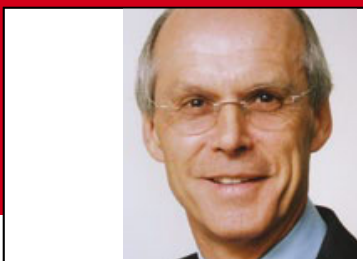

Prof. Dr. med. H. S. FüeßI

Isar-Amper-

Klinikum,

KI. München-

Ost, Haar

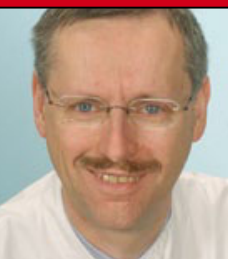

Prof. Dr. med. W. Zidek

Campus Benjamin Franklin, Charité Centrum, Berlin

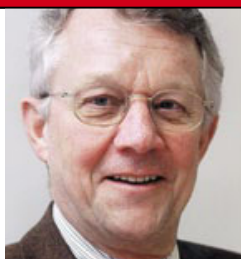

Prof. Dr. med. H. Holzgreve

Internist, Kardiologische Praxis, München

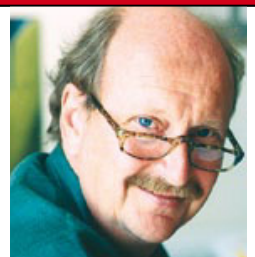

Prof. Dr. med.

E. Ernst

Peninsular

Medical School,

University of Exeter/UK

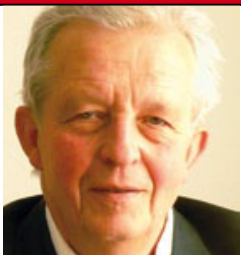

Prof. Dr. med. K. Malberg Immunologie, DresdenLoschwitz

\section{Gesunde Schlafdauer verlangt nach Mittelmaß}

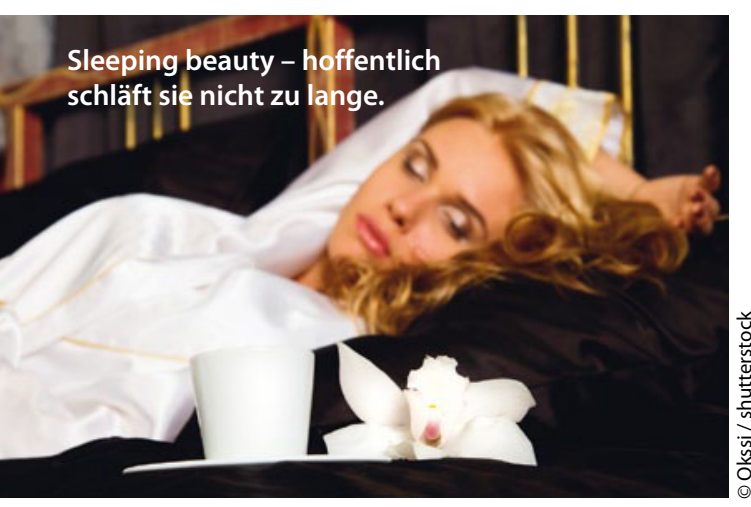

Sowohl zu kurze als auch zu lange Schlafdauer verursacht oder signalisiert ein erhöhtes Risiko für kardiovaskuläre Erkrankungen.

- Die Autoren interessierten sich für den Zusammenhang von Schlafdauer mit tödlichen bzw. nicht tödlichen Komplikationen von koronarer Herzkrankheit, von Schlaganfällen und anderen kardiovaskulären Erkrankungen. Sie entdeckten zu diesem Thema 15 prospektive Studien mit einer Dauer über mindestens drei Jahre. In der Mehrzahl der Studien galten 7-8 Stunden Schlaf als normal, unter 5-6 Stunden als kurz und über 8-9 Stunden als lang. Insgesamt konnten die Daten von 474684 männlichen und weiblichen Probanden mit einer Dauer zwischen 6,9 und 25 Jahren mit 16067 kardiovaskulären Komplikationen auswertet werden.

Sowohl kurze als auch lange Schlafdauer erhöhten jeweils signifikant das Risiko für Komplikationen der koronaren Herzkrankheit und für Schlaganfälle um das 1,15- bis 1,65-Fache. Das trifft auch für die Gesamtzahl aller kardiovaskulären Komplikationen zu, wobei allerdings nur die Korrelation mit langer Schlafdauer Signifikanz erreicht (s. Tab.). Diese Aussagen gelten gleichermaßen für Männer und Frauen, un-

\section{Tabelle 1}

Risiko für kardiovaskuläre Erkrankungen bei kurzer und langer Schlafdauer im Vergleich zur Kontrollgruppe mit 7-8 Stunden Schlaf

\begin{tabular}{l|l|l|l|l|} 
& \multicolumn{2}{|c|}{ kurze Schlafdauer } & \multicolumn{2}{l|}{ lange Schlafdauer } \\
\hline Koronare Herzkrankheit & 1,48 & $\mathrm{P}=0,0001$ & 1,38 & $\mathrm{P}=0,0005$ \\
\hline Schlaganfall & 1,15 & $\mathrm{P}=0,047$ & 1,65 & $\mathrm{P}=0,0001$ \\
\hline Alle kardiovask. Ereignisse & 1,03 & $\mathrm{P}=0,52$ & 1,41 & $\mathrm{P}=0,0001$ \\
\cline { 1 - 3 } & & & & \\
\cline { 1 - 3 } & &
\end{tabular}

terschiedlich lange Beobachtungsdauer und geographische Regionen.

\section{Kommentar}

Zur Frage, wie lange ein gesunder Schlaf dauern soll, gibt es eine Fülle von Untersuchungen und keinesfalls nur übereinstimmende Antworten. Angesichts der Datenfülle dieser Analyse wird wahrscheinlich keine zukünftige Studie das jetzige Ergebnis modifizieren: am besten sind 7-8 Stunden Schlaf, und zu beiden Seiten hin steigt das Risiko für kardiovaskuläre Erkrankungen. Über mögliche physiologische Ursachen bei kurzem Schlaf, z. B. metabolische, hormonelle und inflammatorische Veränderungen wird spekuliert. Die negativen Folgen bei langem Schlaf sind biologisch weniger gut zu erklären, weil auch Merkmale wie Depression, niedriger sozioökonomischer Status, Arbeitslosigkeit, geringe körperliche Aktivität und unerkannte Erkrankungen mit langem Schlaf korrelieren. Es kann also durchaus sein, dass die Schlafdauer nicht nur bzw. nicht ausschließlich ein Risikofaktor, sondern bereits ein frühes Merkmal für latente oder bereits manifeste kardiovaskuläre Erkrankungen ist.

H. HOLZGREVE -

\section{- F. P. Cappuccio et al.}

Sleep duration predicts cardiovascular out-comes: a systematic review and metaanalysis of prospective studies. Eur. Heart J. 32 (2011) doi:10.1093/eurheartj/ehr007 